\title{
Letter to the editor regarding: 'Impalpable breast lesion localisation, a logistical challenge: results of the UK iBRA-NET national practice questionnaire'
}

\author{
Benjamin Patel ${ }^{1}$ (1) $\cdot$ Louise Merker $^{1} \cdot$ Ajay Sahu $^{1}$
}

Received: 23 February 2021 / Accepted: 3 March 2021 / Published online: 13 March 2021

(c) The Author(s), under exclusive licence to Springer Science+Business Media, LLC, part of Springer Nature 2021

To the editor,

Somasundaram and colleagues [1], as part of the iBRANet Localisation Steering Group, recently reported the results of a phase 1 national practice questionnaire of impalpable breast lesion localisations in the UK. This insightful and topical study found that wire-guided localisation (WGL) was the most common localisation technique (82/98 units) and highlighted several frustrations with this technique, including theatre scheduling, wire migration, difficulties locating the tip, higher re-excision rates and poor acceptance to patients. Indeed, the majority (55/98 units) voiced their preference to switch to an alternative technique.

One finding of this study is that localisation is rarely performed by the surgeon. Instead, consultant radiologists and radiographers primarily perform localisation. 65 units noted that 'better use of radiology time' was a reason for changing current localisation practices. In our experience, use of ultrasound by surgeons is an effective adjunct in aiding localisation of both palpable and impalpable lesions, providing key information regarding the size and depth of a lesion at the time of surgery. The majority of impalpable mass lesions are identifiable on ultrasound. For example, in our Breast Unit between 1st May 2020 and 1st August 2020, $45 / 48$ impalpable lesions were visualised on ultrasound. Surgeons who are trained in ultrasound are able to insert wires in theatre on the day of surgery for truly impalpable tumours or undertake ultrasound-guided excision, which itself has been shown to be an effective method for excision of impalpable lesions [2, 3].

In the iBRA-NET questionnaire, 74/98 units cited 'logistical issues' as a limitation to current localisation practices. For example, 60/98 undertook the localisation procedure

Benjamin Patel

benjpatel@gmail.com

1 Southmead Hospital, Bristol, UK in a different part of the hospital to the operation, 17/98 undertook the procedure in a different building and 17/98 undertook the procedure at a different site. There was significant reliance on porters to transfer patients between different parts of the hospital. Not only is this resource-intensive, but it is also a source of unnecessary contact that, during the COVID-19 pandemic, hospitals have been striving to limit.

The logistics of wire insertion result in a delay to theatre schedules. In this questionnaire, the most common delay was 60-90 min, but there was a significant range, making planning of operating lists difficult and reducing the flow of the list. Typically, responders were unable to list patients before 10 am due to delays in the localisation procedure. Furthermore, 39/98 units explained that they had delays at least twice per week and the majority experienced late running of lists on at least a monthly basis due to these delays.

51/98 units cited 'not patient friendly' as a limitation to current localisation practices. Patients are dissatisfied at multiple levels by WGL practices: disruption to their life by undergoing an additional procedure sometimes on a separate day, locating different departments, discomfort during the insertion. In addition, delays between wire insertion and assessment of the patient in theatre can result in wire migration, which was a perceived limitation of current localisation practices for $46 / 98$ units.

If breast surgeons were trained in ultrasound, many of these limitations could be addressed: the logistical challenge would be improved by insertion of wires in theatre, minimising transport between different sites and reducing delays. Patient experience would be improved with wires being inserted under general anaesthetic. Furthermore, surgeons would not have to estimate location of the tip of the wire using two dimensional mammographic images. Instead, having imaged the lesion and inserted the wire themselves, they would have an immediate appreciate for the position in the breast during the operation. Several alternatives to WGL were considered in this study. However, the most common 
barrier to change cited by responders was cost. If surgeons were trained in ultrasound, cost would be saved by reduced radiology resource and reduced need for porters.

The utility of ultrasound to a breast surgeon cannot be underestimated and extends beyond localisation of impalpable lesions. It has applications in clinic for assessing lesions, guiding core biopsy and drainage of abscesses. It is a visual tool that allows an enhanced appreciation of a breast lesion. For these reasons, we argue that ultrasound should be a fundamental part of the breast trainees' curriculum. With the new curriculum changes coming into place this August, we have an opportunity for our future breast surgeons to hone their training more specifically in breast care and basic use of ultrasound should be part of this. This would ultimately improve the patient experience, enhance hospital efficiency, provide cost and time savings and augment a breast surgeon's appreciation of breast lesions.

\section{References}

1. Somasundaram SK et al (2021) Impalpable breast lesion localisation, a logistical challenge: results of the UK iBRA-NET national practice questionnaire. Breast Cancer Res Treat 185(1):13-20. https://doi.org/10.1007/s10549-020-05918-6

2. Ramos M et al (2013) Ultrasound-guided excision combined with intraoperative assessment of gross macroscopic margins decreases the rate of reoperations for non-palpable invasive breast cancer. Breast Edinb Scotl 22(4):520-524. https://doi.org/10.1016/j.breas t.2012.10.006

3. Rahusen FD, Bremers AJA, Fabry HFJ, van Amerongen AHMT, Boom RPA, Meijer S (2002) Ultrasound-guided lumpectomy of nonpalpable breast cancer versus wire-guided resection: a randomized clinical trial. Ann Surg Oncol 9(10):994-998. https://doi. org/10.1007/BF02574518

Publisher's Note Springer Nature remains neutral with regard to jurisdictional claims in published maps and institutional affiliations.

Funding None.

\section{Declarations}

Conflict of interest None. 